\title{
Distintos estilos del sentido del humor y su relación con las conductas agresivas físicas y verbales en adolescentes argentinos
}

\author{
Different styles of sense of humor and their relationship with aggressive \\ physical and verbal behavior in Argentine adolescents
}

Eugenia López ${ }^{1}$, Belén Mesurado² y Paulina Guerra ${ }^{3}$

${ }^{1}$ Psicóloga. Psicóloga Clínica en el Instituto de Neurología Cognitiva (INECO).

E-mail: elopez@ineco.org.ar

\begin{abstract}
${ }^{2}$ Doctora en Psicología. Investigadora Adjunta en el Centro Interdisciplinario de Investigaciones en Psicología Matemática y Experimental (CIIPME), Consejo Nacional de Investigaciones Científicas y Técnicas (CONICET). Profesora de grado en la Universidad Austral.

E-mail: bmesurado@conicet.gov.ar
\end{abstract}

${ }^{3}$ Psicóloga. Becaria Doctoral en el Centro Interdisciplinario de Investigaciones en Psicología Matemática y Experimental (CIIPME), Consejo Nacional de Investigaciones Científicas y Técnicas (CONICET). Profesora de grado de la Universidad Austral.

E-mail: paulinaguerra@conicet.gov.ar

Centro Interdisciplinario de Investigaciones en Psicología Matemática y Experimental (CIIPME). Consejo Nacional de Investigaciones Científicas y Técnicas (CONICET).

Ciudad Autónoma de Buenos Aires. Argentina.

\section{Resumen}

El objetivo de este trabajo es estudiar la relación entre los estilos de humor adaptativos (humor afiliativo y humor mejoramiento personal) y desadaptativos (humor agresivo $y$ humor descalificación personal) propuestos por Martin (2003), y las conductas agresivas físicas y verbales. Se trabajó con una muestra de 100 adolescentes argentinos, entre $16 \mathrm{y}$ 18 años, de ambos sexos, quienes respondieron dos escalas: el Cuestionario de Agresividad Física y Verbal (Caprara y Pastorelli, 1993, versión española de del Barrio, Moreno Rosset, López y Martínez, 2001), y la versión adaptada al español por Cayssials (2004) de la Escala de Estilos de Humor (Martin, 2003). Los resultados indican que el humor agresivo predice positivamente las conductas agresivas en general, tanto verbales como físicas, mientras que el estilo de humor de mejoramiento personal mitiga las conductas agresivas evaluadas en general y las conductas agresivas verbales. Por último, las conductas agresivas físicas únicamente fueron predichas por el estilo de humor agresivo.

Palabras clave: Sentido del humor adaptativo; Sentido del humor desadaptativo; Agresividad fisica; Agresividad verbal; Adolescentes. 


\section{Abstract}

The sense of humor gives a new perspective of life by turning the person into a spectator of what is happening, being able to laugh at oneself and to what happens to one. Because of that, it is considered that humor allows us to face problematic situations in a different way.

Carbelo and Jáuregui (2006) argue that sense of humor is not a mere remedy to prevent or help overcome disease; instead, it has the capacity to promote greater well-being, enjoyment of life, growth towards greater humanity and fulfillment. These same authors add that sense of humor is a unique trait of the human species and it is possessed by all human beings of different cultures (Carbelo y Jáuregui, 2006). While each culture has its own rules about which facts or situations may be the object or cause of humor, "both humor and laughter are universal" (Cassaretto y Martínez, 2009, p. 289). Although humor has been associated with psychological, physical and social well-being, not all research show consistent results. This is because sense of humor does not imply only positive components, but also negative components (Martin, 2003; Cassaretto y Martínez, 2009).

In general, the different studies carried out have perceived sense of humor as a positive feature with the potential to generate positive effects. However, there are negative elements of humor that have a negative correlation with psychological well-being (Martin, 2003; Grimshaw, Kirsh, Kuiper y Leite, 2004). Therefore, it is important to be able to consider the sense of humor not as a one-dimensional construct (taking into account only its positive components), but to be able to focus on a multi-dimensional approach capable of considering all the characteristics and consequences - positive and negative- of it. Martin (2003) postulates that humor is a personality trait composed of either healthy or adaptive components and of unhealthy or unadaptable components. This author proposes the following classification: Adaptive humor is formed by affiliative humor (the tendency to say funny things, to make jokes, and spontaneous and witty jokes to amuse other people) and self-enhancing humor (it implies having fun with the incongruities of life even in adversity and using humor as a strategy to face the vicissitudes), and unadaptive humor is formed by aggressive humor (the tendency to use humor for the purpose of criticizing or manipulating others by resorting to sarcasm, bothering with jokes, ridiculing others) and self-defeating humor (people who use this kind of humor are self-deprecating, make or say funny things at the expense of themselves and laugh with others while ridiculing or belittling). The aim of this work is to study the relationship between these two types of humor and physical and verbal aggressive behaviors. We worked with a sample of 100 adolescents from Argentina of both sexes, aged between 16 and 18 years, of the middle socioeconomic strata of Buenos Aires, Argentina. All participants responded to two scales: the questionnaire of physical and Verbal aggression (Caprara y Pastorelli, 1993, Spanish version of Barrio, Moreno Rosset, López Martínez, 2001), and the version adapted to Spanish by Cayssials (2004) of the scale of humor styles (Martin, 2003). The results indicate that aggressive humor positively predicts aggressive behaviors evaluated in a general way, and aggressive behaviors verbal and physical evaluated specifically. On the other hand, personal improvement humor style mitigates general aggressive behaviors and verbal aggression specifically. Finally, aggressive physical behaviors were only predicted by the aggressive humor style.

Keywords: Adaptive sense of humor; Unadaptive sense of humor; Physical aggression; Verbal aggression; Adolescents. 


\section{Introducción}

A lo largo de la historia se habló y teorizó acerca del humor desde la Teoría de los Humores de Hipócrates; sin embargo, recién en los últimos 20 años se ha puesto énfasis en el estudio científico del sentido del humor (Martin, 2003). El sentido del humor brinda una nueva perspectiva acerca de la vida, ubicando a la persona como un expectante de lo que ocurre, pudiendo reírse de sí mismo y/o de lo que le sucede; de aquí que se considere que el humor permite afrontar las situaciones problemáticas de un modo diferente. Allport (1965) considera al humor como una de las características fundamentales de la personalidad madura que le da la capacidad de reírse de las cosas. Por otro lado, sostiene que el reírse de uno mismo puede ser el camino para autogestionar la cura del neuroticismo (Allport, 1950). En esta misma línea, Carbelo y Jáuregui (2006) sostienen que el sentido del humor no se trata de un mero remedio para prevenir o ayudar a superar la enfermedad, sino de una capacidad que fomenta un mayor bienestar y disfrute de la vida, el crecimiento hacia una mayor humanidad y plenitud. Estos mismos autores agregan que el sentido del humor es una capacidad única del hombre $\mathrm{y}$ que la poseen todos los seres humanos de diferentes culturas (Carbelo y Jáuregui, 2006). Si bien cada cultura tiene sus propias normas acerca de qué hechos o situaciones pueden ser objeto o causa de humor, "tanto el humor como la risa son universales" (Cassaretto y Martínez, 2009). El sentido del humor "puede aludir a características del estímulo, a los procesos mentales presentes en la creación, percepción, comprensión y apreciación de situaciones humorísticas o a las respuestas que da un sujeto determinado frente a situaciones diversas" (Cayssials, 2004). Y, si bien se trata de un fenómeno que se manifiesta en un espacio interpersonal, puede llegar a ser exclusivamente intrapsíquico.

Investigaciones previas han mostrado que al sentido del humor se le han atribuido muchos beneficios, tanto psicológicos como físicos y sociales (Carbelo y Jáuregui, 2006). En cuanto a los efectos psicológicos, en un estudio realizado en pacientes con demencia, se mostró que el humor adaptativo estuvo positivamente relacionado con un mayor sentido de vida, dando la posibilidad a estos pacientes de mejores conexiones sociales con los otros (Mak, Sörensen y Pruchno, 2018). El humor es además capaz de reducir el estrés y la ansiedad, ayuda a prevenir la depresión y a superar momentos de adversidad (Carbelo y Jáuregui, 2006; Fritz, Russek y Dillon, 2017; Grimshaw, Kirsh, Kuiper y Leite, 2004). Asimismo, el sentido del humor está asociado al autoconcepto positivo y a una buena autoestima (Grimshaw et al., 2004). En relación a los efectos del humor en la salud física, se ha demostrado que ayuda a lograr la relajación muscular $y$, por ende, a tener una mejor respiración, así como también a tolerar el dolor durante más tiempo (Abel, 2002). Por un lado, estimula la liberación de endorfina, que además de ser un analgésico natural, es una hormona que brinda una sensación de bienestar, favorece a la producción de inmunoglobulina A y ayuda a mantener en funcionamiento la glándula timo, encargada de madurar los linfocitos brindando inmunidad al organismo. Por otro lado, disminuye la hormona suprarrenal cortisol, que aumenta frente a situaciones estresantes (García Pintos, 2009). También genera beneficios en el área social, ya que aquellas personas que tienen un gran sentido del humor suelen ser más competentes y asertivos socialmente, lo cual produce relaciones sociales más cercanas y satisfactorias (Carbelo y Jáuregui, 2006).

Si bien se han demostrado todas estas asociaciones positivas entre el humor y el bienestar psicológico, físico y social, no todas las investigaciones muestran efectos positivos del humor. Esto se debe a que el sentido del humor no implica solo componentes positivos, sino también negativos (Martin, 2003; Cassaretto y Martínez, 2009). Por lo general, en las distintas investigaciones realizadas se 
ha considerado al sentido del humor como una característica positiva con potencial para generar efectos positivos. Sin embargo, existen elementos negativos del humor que poseen una correlación negativa con el bienestar psicológico (Martin, 2003; Grimshaw et al., 2004). Por ello, es importante poder considerar al sentido del humor no como un constructo unidimensional (teniendo en cuenta solo sus componentes positivos), sino poder centrarse en un enfoque multidimensional capaz de considerar todas sus características y consecuencias positivas y negativas. Martin (2003) postula que el humor es un rasgo de personalidad compuesto tanto por componentes saludables o adaptativos como por componentes no saludables o desadaptativos. Este autor propone la siguiente clasificación sobre los tipos de humor:

1.- Afiliativo (Affiliative): Es la tendencia a decir cosas divertidas, a hacer chistes y bromas, burlas espontáneas e ingeniosas para divertir a otras personas. Este estilo de humor involucra bromear con los demás, contar historias divertidas y reír con otros en un esfuerzo por promover las relaciones con los demás o amortiguar la tensión interpersonal. Se trata de un estilo esencialmente carente de hostilidad y su uso ubica adecuadamente tanto a quien lo utiliza como a los demás.

2.- Mejoramiento personal (Self-enhancing): Se refiere a la tendencia a mantener una perspectiva humorística de la vida aunque ésta no sea compartida con otros. Implica divertirse con las incongruencias de la vida aun en la adversidad y usar el humor como una estrategia para afrontar las vicisitudes. Se usa dicho humor como una estrategia de afrontamiento para regular las emociones negativas y afrontarlas de forma que la persona no se dañe a sí mima ni a otros.

3.- Agresivo (Aggressive): Es la tendencia a usar el humor con el propósito de criticar o manipular a otros recurriendo al sarcasmo, molestando con bromas, poniendo en ridí- culo a los demás. Incluye los esfuerzos por sobresalir a expensas de la relación con los otros. Es una forma potencialmente ofensiva del humor. En este caso el humorista se impone a expensas de los demás.

4.- Descalificación personal (Self-defeating): Las personas que usan este tipo de humor son autodespreciativos, hacen $\mathrm{o}$ dicen cosas divertidas a expensas de sí mismos y se ríen con otros al tiempo que se ridiculizan o menosprecian. Implica también el uso del humor como una forma negativa de defensa para ocultar sus sentimientos. Este estilo de humor parece ser un intento de congraciarse consigo mismo o de ganar la aprobación de los otros a expensas de sí mismo.

Cabe destacar, que tanto el sentido del humor afiliativo como el agresivo se focalizan en el uso del humor en la relación interpersonal. Por un lado, el primero contribuiría a forjar nuevas relaciones interpersonales y a manternerlas en el tiempo, mientras que el humor agresivo socavaría estas relaciones (Yip y Martin, 2006). Por otro lado, el mejoramiento personal y el estilo de descalificación personal, se centran en el uso del humor hacia uno mismo. Es decir, que se pueden clasificar estos estilos de acuerdo a cómo se expresa el humor (a los demás o a sí mismo). Así, de los cuatro estilos nombrados, dos aluden al humor como una actividad relacional y los otros hacen referencia al sentido del humor como una actividad intrapsíquica (Martin, 2003; Grimshaw, Kirsh, Kuiper y Leite, 2004).

Asimismo, los tipos de humor afiliativo y mejoramiento personal podrían estar asociados a la salud mental y a la satisfacción con la vida, por lo que se denominan estilos adaptativos, mientras que los dos restantes (agresivo y descalificación personal) mostraron estar asociados con distintas sintomatologías psicopatológicas como depresión, ansiedad, trastornos de personalidad límite, hostilidad, malicia y soledad, por lo que se denominan desadaptativos (Brizzio, Carreras y Casullo, 2006; Grimshaw et al., 2004; Martin, Brinker, 
Frewen y Dozois, 2008; Meyer et al., 2017; Salavera y Usán 2017; Schermer et al., 2017; Vrabel, Zeigler-Hill y Shango, 2017).

En base a lo formulado, el objetivo de esta investigación fue analizar la relación entre los estilos de humor tanto adaptativos como desadaptativos y las conductas agresivas (tanto verbales como físicas) en adolescentes. Se formuló la siguiente hipótesis: el humor adaptativo inhibe las conductas agresivas en general así como las conductas agresivas, tanto físicas como verbales, mientras que el humor desadaptativo promueve las conductas agresivas en general así como la agresión física y verbal.

\section{Metodología}

\section{Muestra}

El estudio es de alcance correlacional, de diseño transversal y la selección de la muestra fue intencional no probabilística (Hernández Sampieri, Fernández Collado y Baptista Lucio, 2010). La muestra estuvo compuesta por 100 adolescentes, varones y mujeres, de 16 a 18 años $(\mathrm{M}=17.24 ; \mathrm{SD}=.57)$, todos ellos alumnos de 4to y 5to año de colegios secundarios de la ciudad de Buenos Aires, Argentina. El $60 \%$ fueron mujeres $(\mathrm{n}=60)$ y el $40 \%$ fueron varones $(n=40)$, todos de nivel socioeconómico medio.

\section{Procedimiento}

Las evaluaciones fueron autoadministradas en grupos de aproximadamente 15 adolescentes, de forma voluntaria y anónima, contando con las debidas autorizaciones de los padres y el consentimiento de los alumnos que participaron del estudio. El tiempo aproximado de las evaluaciones fue de 20 minutos. Los alumnos que participaron en el estudio recibieron los cuestionarios con buena disposición respondiendo a las evaluaciones sin dificultad y mostrándose interesados en completar las escalas.

\section{Instrumentos}

\section{Escala de Estilos de Humor (Martin y Doris, 2003).}

Es un instrumento que evalúa los 4 estilos del sentido del humor. Es autoadministrable y consiste en 32 ítems, 8 para cada una de las 4 subescalas correspondientes a los distintos estilos de humor (Afiliativo, Mejoramiento Personal, Agresivo y Descalificación Personal). Se la puede administrar tanto a adultos como a adolescentes. En este estudio se utilizó la versión adaptada por Cayssials (2004) para adolescentes de población argentina. Esta versión tiene un coeficiente Alfa de Cronbach general de .75. Las subescalas obtuvieron niveles de confiabilidad adecuados: Afiliativo: $\alpha=.65$; Mejoramiento Personal: $\alpha=.71$; Agresividad: $\alpha=.72$ y Descalificación Personal: $\alpha=.60$.

\section{Cuestionario de Agresividad Física y Verbal.}

Es un instrumento para la evaluación de la agresión física y verbal en niños y adolescentes. Fue diseñado por Caprara y Pastorelli (1993) y luego adaptado al español por del Barrio, Moreno Rosset y López Martínez (2001). Consiste en 20 ítems que describen conductas agresivas tanto físicas como verbales, acompañados de una escala graduada en tres niveles de frecuencia: 3 (A menudo), 2 (Algunas veces), 1 (Nunca). De estos 20 ítems, 5 son distractores y se excluyen al obtener la puntuación de agresividad. Los análisis de confiabilidad muestran un Alfa de Cronbach de .80 para el puntaje total, .85 para la agresión verbal y .79 para la subescala agresión física.

\section{Procedimiento estadístico.}

Luego de administrar las escalas, con los datos obtenidos se diseñó una base mediante el programa estadístico Statistical Packge for the Social Science (SPSS) para realizar un análisis descriptivo de los mismos y un 
estudio de correlación entre las variables en estudio. Posteriormente se realizó el análisis de regresión lineal con el objetivo de estudiar el poder predictivo de los distintos tipos de humor sobre la agresividad en general y por último, se repitió el análisis con cada uno de los tipos de agresividad física y verbal.

\section{Resultados}

\section{Análisis descriptivo de los datos}

En la Tabla 1 se informan las medias y los desvíos estándar de cada una de las variables incluidas en el estudio.

Tabla 1. Análisis descriptivo de los datos $N=100$.

\begin{tabular}{ccc}
\hline Variables & Medias & Desvío Estándar \\
\hline Humor & & \\
Afiliativo & 5.70 & 0.82 \\
Mejoramiento personal & 4.48 & 0.89 \\
Agresivo & 3.50 & 1.05 \\
Descalificación personal & 2.98 & 0.79 \\
Agresividad & & \\
Física & 9.15 & 1.99 \\
Verbal & 13.83 & 2.55
\end{tabular}

\section{Relación entre estilos de humor y agresividad}

Los estudios de correlación indicaron que existe una correlación positiva moderada entre el humor agresivo y los distintos tipos de agresividad. Asimismo, los resultados indican que el humor de descalificación personal presenta baja asociación con la agresividad cuando se la considera en su totalidad y con la agresividad física en particular, sin presentar asociación alguna con la agresividad verbal.

En cuanto a los estilos de humor adaptativos, el tipo mejoramiento personal presenta una correlación negativa baja con la agresividad verbal. Por el contrario, el humor afiliativo no presenta relación con la agresividad en ninguna de sus categorías (ver Tabla 2).

Tabla 2. Correlación entre los estilos de humor y la agresividad $(N=100)$.

\begin{tabular}{cccc}
\hline \multirow{2}{*}{ Estilos de humor } & \multicolumn{3}{c}{ Agresividad } \\
\cline { 2 - 4 } & Física & Verbal & Total \\
\hline Afiliativo & .071 & .079 & .083 \\
Mejoramiento Personal & -.112 & $-.204^{*}$ & $-.178^{*}$ \\
Agresivo & $.512^{* *}$ & $.633^{* *}$ & $.632^{* *}$ \\
Descalificación Personal & $.234^{*}$ & .170 & $.216^{*}$ \\
\hline
\end{tabular}

Nota: ${ }^{*} \mathrm{p}<.05,{ }^{* *} \mathrm{p}<.01$. 


\section{Predicción de la agresividad}

Con la intención de poner a prueba la hipótesis de trabajo, se realizaron los tres estudios de regresión analizando la influencia de los diferentes tipos de humor adaptativos y desadaptativos (VI) sobre diferentes aspectos de la agresión (VD).

Primero, se realizó un Análisis de Regresión Lineal con el objetivo de estudiar si los distintos tipos de humor permiten predecir la agresividad en general. Los resultados indican que el modelo fue significativo $[F(4,99)=17.97, \mathrm{p} \leq .001]$, el humor agresivo predice positivamente la agresividad considerada en general $(\beta=.599)$ mientras que el estilo de humor de mejoramiento predice negativamente la agresividad ( $\beta=-.171)$ (ver Tabla 3). Estos análisis de regresión explicaron el $43 \%$ de la varianza.

Tabla 3. Predicción de la agresividad total $(N=100)$.

\begin{tabular}{cccc}
\hline Variables & B & EE C & $\beta$ \\
\hline Humor afiliativo & .008 & .030 & .024 \\
Humor mejoramiento personal & -.053 & .027 & $-.171^{*}$ \\
Humor agresivo & .158 & .022 & $.599 * * *$ \\
Humor descalificación personal & .032 & .029 & .090 \\
\hline
\end{tabular}

Nota: ${ }^{*} \mathrm{p}<.05, * * * \mathrm{p}<.001$.

Seguidamente se realizaron otros dos Análisis de Regresión Lineal para estudiar si los distintos estilos de humor predecían de modo diferente los distintos tipos de agresividad verbal y física. Los modelos fueron significativos tanto para predecir la agresión verbal $[\mathrm{F}(4,99)=18.26, \mathrm{p} \leq .001]$, como la física $[\mathrm{F}(4,99)=9.54, \mathrm{p} \leq .001]$.

Los resultados indican que tanto el humor agresivo como el tipo de humor mejoramiento personal permiten predecir la agresividad verbal; el primero lo hace positivamente ( $\beta=$ .606) mientras que el segundo lo hace negativamente $(\beta=-.199)$. Es decir que el mejoramiento personal protege al adolescente de desarrollar conductas agresivas verbales (ver Tabla 4). Estos análisis de regresión explicaron el $44 \%$ de la varianza.

Tabla 4. Predicción de la agresividad verbal $(N=100)$.

\begin{tabular}{cccc}
\hline Variables & B & EE C & $\beta$ \\
\hline Humor afiliativo & .131 & .279 & .042 \\
Humor mejoramiento personal & -.570 & .250 & $-.199^{*}$ \\
Humor agresivo & 1.473 & .197 & $.606^{* * *}$ \\
Humor descalificación personal & .134 & .262 & .041 \\
\hline
\end{tabular}

Nota: $* \mathrm{p}<.05, * * * \mathrm{p}<.001$.

Sin embargo, cuando se analizó el poder predictivo de los estilos de humor sobre la agresividad física, solamente el humor agre- sivo surge como predictor positivo $(\beta=.476)$ (ver Tabla 5). Estos análisis de regresión explicaron el $29 \%$ de la varianza. 
Tabla 5. Predicción de la agresividad física $(N=100)$.

\begin{tabular}{cccc}
\hline Variables & B & EE C & $\beta$ \\
\hline Humor afiliativo & -.007 & .244 & -.003 \\
Humor mejoramiento personal & -.232 & .219 & -.104 \\
Humor agresivo & .901 & .173 & $.476^{* * *}$ \\
Humor descalificación personal & .341 & .229 & .135 \\
\hline
\end{tabular}

Nota: $* \mathrm{p}<.05, * * * \mathrm{p}<.001$.

\section{Discusión}

El objetivo de la presente investigación fue estudiar la relación entre el humor adaptativo y desadaptativo con la agresión evaluada de forma global y diferenciando distintos aspectos de la agresión física y verbal. Los resultados indican que la hipótesis planteada pudo comprobarse sólo parcialmente y que los diferentes tipos de humor juegan un rol variado en la relación o predicción de las conductas agresivas, por lo que es importante distinguirlas.

Los resultados obtenidos mostraron una correlación positiva y significativa entre el humor agresivo - estilo de humor desadaptativo- y la agresividad evaluada de forma global. Esto coincide con estudios previos que muestran que el humor desadaptativo está asociado con diferentes sintomatologías psicopatológicas (Grimshaw et al., 2004; Brizzio et al., 2006; Meyer et al., 2017; Schermer et al., 2017; Vrabel et al., 2017).

Dentro de estos estilos desadaptativos del humor, cabe destacar que el estilo de humor agresivo resultó ser el estilo más fuertemente relacionado con la agresión tanto física como verbal. Estos resultados son consistentes con la teoría que sustenta estos estudios; ya Martin (2003) describió el humor agresivo como una forma potencialmente ofensiva del humor. Entre otras cosas implica criticar, manipular, utilizar el sarcasmo, molestar y ridiculizar a los otros, con la intención de sobresalir a expensas de los demás. Podría pensarse entonces que, aquel que posee un estilo de humor agresivo podría utilizar con mayor frecuencia la agresividad tanto física como verbal para imponerse sobre los demás.

En cuanto al estilo de humor de descalificación personal -el otro estilo de humor desadaptativo- no se halló relación con la agresividad evaluada de forma global, ni con la agresividad física y verbal. Este estilo consiste en hacer o decir cosas divertidas a expensas de sí mismo, auto-ridiculizarse y dejar que los demás se rían de uno. Es probable que los adolescentes que usen este tipo de humor lo hagan con la intención de ser aceptados en un grupo, lo que puede conducir a problemas más internalizantes (ej. la depresión) que externalizantes en los adolescentes, de allí que no se encontró relación con las conductas agresivas. En otras palabras, este tipo de humor puede estar más vinculado a problemas caracterizados por la rumiación de pensamientos negativos sobre sí mismo, sin verse reflejado en conductas disruptivas hacia otros.

En segundo lugar, se encontraron diferencias en la relación de los estilos de humor adaptativos y la agresividad en sus distintas categorías. El estilo afiliativo no se relacionó con ningún tipo de agresividad. Esto coincide con lo postulado por Martin (2003), quien plantea que el estilo afiliativo de humor carece esencialmente de hostilidad. Por otro lado, se manifestó una correlación negativa entre el estilo mejoramiento personal y la agresividad verbal. Pareciera que tener una visión humorística de las adversidades de la vida, 
tener buen humor -aunque uno no esté bien-, y usar el humor para sentirse mejor cuando uno está enojado, contribuye a manejar mejor las conductas agresivas verbales. El humor de mejoramiento personal implica que se posee la capacidad de reírse ante la adversidad y enfrentar las vicisitudes de la vida promoviendo cambios positivos (Boerner y Murphy, 2017), al mismo tiempo que permite regular las emociones negativas evitando lastimarse a sí mismo y a los demás (Martin, 2003). En otras palabras, el mejoramiento personal funcionaría como factor protector evitando la aparición de la agresividad verbal, reemplazándolas por bromas y risas. Esto va en la misma dirección de lo hallado en investigaciones anteriores, en las que el mejoramiento personal se relacionó negativamente con la aparición de sintomatología psicopatológica (Brizzio et al., 2006; Grimshaw et al., 2004).

Por último, es importante destacar que en los análisis de regresión lineal realizados en este estudio se obtuvieron niveles elevados de variancia explicada en todos los modelos analizados. En concreto, en relación a la predicción del humor de la agresividad evaluada de forma global, se obtuvo una varianza explicada del $43 \%$, con la agresividad verbal una varianza explicada de $44 \%$ y con la agresividad física una varianza de $29 \%$, lo cual alerta sobre la importancia de la expresión del humor en la predicción de las conductas agresivas.

Sin embargo, es importante resaltar que una de las mayores debilidades del presente estudio es la representatividad de la muestra; por otro lado el número de participantes es bajo, de allí que futuros estudios serán necesarios para confirmar estos resultados.

\section{Referencias bibliográficas}

Allport, G.W. (1965). Psicología de la personalidad. Buenos Aires: Paidós.

Allport, G.W. (1950). The individual and his religion. New York: Macmillan.
Abel, M. (2002). Humor, stress and coping strategies. Humor: International Journal of Humor Research, 15(4), 365-381. doi: 10.1515/ humr.15.4.365

Boerner, M., Joseph, S.

y Murphy, D. (2017). The association between sense of humor and trauma-related mental health outcomes: Two exploratory studies. Journal of Loss and Trauma, 22(5), 440-452. doi: 10.1080/15625024.2017.1310504

Brizzio, A., Carreras, A. y Casullo, M. (2006). Sentido del humor y sintomatología psicopatológica: Un estudio con adolescentes y adultos jóvenes argentinos. Revista del Instituto de Investigaciones de la Facultad de Psicología, 1, 7-21.

Caprara, G.V. y Pastorelli, C. (1993). Early emotional instability, prosocial behavior, and aggression: some methodological aspects. European Journal of Personality, 7, 19-36. doi: 10.1002/per.2410070103

Carbelo, B. y Jáuregui, E. (2006). Emociones positivas: Humor positivo. Papeles del Psicó$\log 0,27,18-30$.

Cassaretto, M. y Martínez, P. (2009). Validación del Sentido del Humor en estudiantes universitarios. Pontificia Universidad Católica del Perú. Revista de Psicología, 27(2), 287-309.

Cayssials, A. (2004). Ficha de cátedra: Sentidos del Humor. (1-4): Facultad de Psicología. Universidad de Buenos Aires.

del Barrio, V.D., Moreno Rosset, C. y López Martínez, R. (2001). Evaluación de la agresión y la inestabilidad emocional en niños españoles: su relación con la depresión. Clínica y Salud, 12(1), 33-50.

Fritz, H.L., Russek, L.N. y Dillon, M.M. (2017). Humor use moderates the relation of stressful life events with psychological distress. Personality and Social Psychology Bulletin, 43(6), 845-859. doi: 10.1177/0146167217699

Grimshaw, M., Kirsh, G., Kuiper, N. y Leite, C. (2004). Humor is not always the best medicine: Specific components of sense of humor and psychological well-being. Humor: Inter- 
national Journal of Humor Research, 17, 135-168. doi: 10.1515/humr.2004.002

Hernández Sampieri, R., Fernández Collado, C. y Baptista Lucio, P. (2010). Metodología de la investigación (Vol. 3). México: McGraw-Hill.

Mak, W., Sörensen, S. y Pruchno, R. (2018). Are humor styles of people with dementia linked to greater purpose in life? The Gerontologist. doi: 10.1093/geront/gnx207

Martin, R. (2003). Sense of humor. En S. López y C.R. Snyder (Eds.), Positive Psychological Assessment. A handbook of models and measures (313-326). Washington, D.C.: American Psychological Association.

Martin, R., Brinker, J., Frewen, P. y Dozois, D. (2008). Humor styles and personality-vulnerability to depression. Humor: International Journal of Humor Research, 21(2), 179-195. doi: 10.1016/j.jrp.2005.08.005

Meyer, N.A., Helle, A.C., Tucker, R.P., Lengel, G.J., DeShong, H.L., Wingate, L.R. y MullinsSweatt, S.N. (2017). Humor styles moderate borderline personality traits and suicide ideation. Psychiatry Research, 249, 337-342. doi: 10.1016/j.psychres.2017.01.038
García Pintos, C. (2009). Sentido del humor. Terapéutica para la vida. En V. Sáenz (Ed.), Logoterapia en acción. Aplicaciones prácticas. Buenos Aires: San Pablo.

Salavera, C. y Usán, P. (2017). Relación entre los estilos del humor y la satisfacción con la vida en estudiantes de Secundaria. European Journal of Investigation in Health, 7(2), 87-97. doi: 10.1989/ejihpe.v7i2.197

Schermer, J.A., Martin, R.A., Vernon, P.A., Martin, N.G., Conde, L.C., Statham, D. y Lynskey, M.T. (2017). Lonely people tend to make fun of themselves: A behavior genetic analysis of humor styles and loneliness. Personality and Individual Differences, 117, 71-73. doi: 10.1016/j.paid.2017.05.042

Vrabel, J.K., Zeigler-Hill, V. y Shango, R.G. (2017). Spitefulness and humor styles. Personality and Individual Differences, 105, 238-243. doi: 10.1016/j.paid.2016.10.001

Yip, J.A. y Martin, R.A. (2006). Sense of humor, emotional intelligence, and social competence. Journal of Research in Personality, 40(6), 1202-1208. doi: 10.1016/j. jrp.2005.08.005

Recibido: 8 de noviembre de 2017 Aceptado: 6 de septiembre de 2019 